\title{
Interkingdom Cross-Talk in Times of Stress: Salmonella Typhimurium Grown in the Presence of Catecholamines Inhibits Porcine Immune Functionality in vitro
}

\author{
Lena Reiske ${ }^{1 *}$, Sonja S. Schmucker ${ }^{1}$, Julia Steuber ${ }^{2}$, Charlotte Toulouse ${ }^{2}$, \\ Birgit Pfaffinger ${ }^{1}$ and Volker Stefanski ${ }^{1 *}$
}

${ }^{1}$ Behavioral Physiology of Livestock, Institute of Animal Science, University of Hohenheim, Stuttgart, Germany, ${ }^{2}$ Cellular Microbiology, Institute of Biology, University of Hohenheim, Stuttgart, Germany

OPEN ACCESS

Edited by:

Reinaldo B. Oria,

Federal University of Ceará, Brazil

Reviewed by:

David Haschka,

Innsbruck Medical University, Austria

lan Antheni Myles,

National Institutes of Health (NIH),

United States

*Correspondence:

Lena Reiske

lena.reiske@uni-hohenheim.de

Volker Stefansk

volker.stefanski@uni-hohenheim.de

Specialty section:

This article was submitted to

Nutritional Immunology,

a section of the journal

Frontiers in Immunology

Received: 13 July 2020

Accepted: 02 September 2020

Published: 30 September 2020

Citation:

Reiske L, Schmucker SS, Steuber J, Toulouse C, Pfaffinger B and Stefanski V (2020) Interkingdom

Cross-Talk in Times of Stress:

Salmonella Typhimurium Grown in the Presence of Catecholamines Inhibits Porcine Immune Functionality in vitro. Front. Immunol. 11:572056.

doi: 10.3389/fimmu.2020.572056
In stressful situations, catecholamines modulate mammalian immune function, and in addition, they can be sensed by many bacteria. Catecholamine sensing was also found in the zoonotic gut pathogen Salmonella Typhimurium, probably contributing to the stress-induced increased risk of salmonellosis. Virulence traits such as proliferation and invasiveness are promoted upon bacterial catecholamine sensing, but it is unknown whether $S$. Typhimurium may also inhibit mammalian immune function in stressful situations. We thus investigated whether supernatants from $S$. Typhimurium grown in the presence of catecholamines modulate porcine mitogen-induced lymphocyte proliferation. Lymphocyte proliferation was reduced by supernatants from catecholamine-exposed Salmonella in a dose-dependent manner. We further examined whether adrenaline oxidation to adrenochrome, which is promoted by bacteria, could be responsible for the observed effect, but this molecule either enhanced lymphocyte functionality or had no effect. We could thereby exclude adrenochrome as a potential immunomodulating agent produced by $S$. Typhimurium. This study is the first to demonstrate that bacteria grown in the presence of catecholamine stress hormones alter their growth environment, probably by producing immunomodulating substances, in a way that host immune response is suppressed. These findings add a new dimension to interkingdom signaling and provide novel clues to explain the increased susceptibility of a stressed host to Salmonella infection.

Keywords: Salmonella Typhimurium, catecholamines, adrenaline, adrenochrome, pig, stress, interkingdom signaling, immune function

\section{INTRODUCTION}

In acute stress situations, the mammalian body launches a rapid physiologic response, which enables it to cope with threats imposed on its health. In the course of such a "fight-orflight" reaction, substantial amounts of stress hormones, particularly adrenaline (ADR) and noradrenaline (NA), can be released from the adrenal gland and at sympathetic nerve endings. These catecholamines (CAs) not only exert effects on blood circulation, respiration, energy metabolism, and many other functions supporting physical exertion (1-3), but also affect the 
immune system $(4,5)$. The long-held view of general immunosuppression by stress hormones was increasingly challenged in recent years, as especially CA actions are rather diverse and dose-dependent, including both inhibiting and enhancing actions (5-9). In some organs, such as the spleen or the gut, stress-related CA release can lead to local concentrations of up to $10^{-4}$ to $10^{-3} \mathrm{M}(10,11)$, which is much higher than in the blood, where levels are between $10^{-9}$ and $10^{-6} \mathrm{M}$ (12, 13). This is caused by NA discharge from synaptic vesicles at noradrenergic nerve endings $(10,11,14)$. In the gut and other tissues with contact to the external world via epithelial surfaces, CAs can even cross the epithelial border and interact with microorganisms living in those ecological niches (15-18). In the colon, NA can reach a concentration of about $50 \mathrm{ng} / \mathrm{g}$ luminal content (14).

In the last two decades, more and more studies in the field of microbial endocrinology emerged, investigating the crosstalk between the endocrine and nervous system of host species and microorganisms inhabiting or invading them. A plethora of microorganisms exist naturally as commensals, e.g., in the gut, oral cavity, and on the skin (19-21). It is therefore no surprise that both parties evolved mechanisms to communicate with each other via mammalian hormones and hormone-like microbial molecules, with mutual benefits supporting symbiosis. However, many pathogens have been proven to sense stressful situations with high CA levels and exploit them by boosting virulence $(22,23)$. NA can be used by many bacterial species as an iron donor $(24,25)$ or activate quorum sensing-a bacterial cell-to-cell communication-by directly binding to QseC or QseE (26-28). Elevated ADR and NA concentrations can thus lead to an increased bacterial growth rate $(29,30)$, motility (26, 29), or attachment to epithelial surfaces (22)-in short, higher chances of infection. This interkingdom signaling works in both directions. Independently of host stress, bacteria produce molecules for interbacterial communication, some of which have a hormone-like side effect on host cells (31). For instance, many Gram-negative bacteria produce substances, which are chemically analogous to eukaryotic lipid hormones and can modulate host immune functions such as neutrophil chemotaxis and lymphocyte proliferation (32-35). Moreover, some quorumsensing molecules produced by several regular inhabitants of the gastrointestinal tract (GIT) probably act as agonists at adrenergic receptors (ARs) (36).

Regarding this intense cross-talk between kingdoms, it is conceivable that in stressful situations, pathogens not only modulate their own properties but may even actively manipulate immune cells to exploit a weakened host. Upon CA perception, they might react with the release of bacterial hormone-like molecules similar to the aforementioned ones. Furthermore, a microbial alteration of mammalian CAs might lead to the formation of an immunomodulating substance. CAs are vulnerable to oxidation (37), and in the presence of superoxide, the oxidation of ADR to adrenochrome (AC) is promoted (38). A boost of AC formation by superoxide-producing bacteria might cause immunomodulation as it was shown that $\mathrm{AC}$ can bind to $\beta$-ARs (39), which can be found on most immune cells (40). Indeed, it was demonstrated in Vibrio cholerae O395N1 that the bacterial $\mathrm{Na}^{+}$-translocating $\mathrm{NADH}$ :quinone oxidoreductase (NQR) promoted the oxidation of ADR to AC by superoxide production (41). AC supported the pathogenicity of $V$. cholerae by stimulating its growth even stronger than ADR and in addition exerted immunomodulating effects by inhibiting tumor necrosis factor $\alpha(\mathrm{TNF}-\alpha)$ production in a human monocytic cell line (41). It can be hypothesized that $V$. cholerae is not the only gut pathogen capable of this reaction, and the promotion of AC formation may be a strategy also used by other bacteria to manipulate host immune functionality. An interesting candidate to test this hypothesis is the important zoonotic gut pathogen, Salmonella enterica ssp. enterica serovar Typhimurium (S. Typhimurium), which is common in domestic pigs (Sus scrofa domestica) and difficult to eradicate. It is known that stress has a negative impact on primary Salmonella infection in pigs and also on the recrudescence of asymptomatic latent infections, for example, by transportation to the slaughterhouse (42). The resulting bacterial shedding by slaughter pigs leads to increased carcass contamination and thus intensifies the risk of food-borne transmission to humans (43). However, despite the importance of this bacterial infection both from a veterinary and a medical point of view, the underlying mechanisms of these observations are still not sufficiently resolved. Because an enhanced motility and growth rate upon CA sensing have also been found in Salmonella $(16,26)$, studying interkingdom signaling is a promising approach to better explain the promotion of salmonellosis by stress.

The aim of the present study was thus to investigate whether $S$. Typhimurium grown in the presence of CAs has the potential to hamper porcine immune functionality. We examined the effects of supernatants from $S$. Typhimurium cultures exposed to NA or ADR on lymphocyte proliferation and demonstrated an inhibitory effect. Furthermore, we investigated whether AC is the causative agent of this inhibition.

\section{MATERIALS AND METHODS}

\section{Animals and Sampling}

To obtain blood for in vitro studies without stress hormone release during the sampling procedure, 37 castrated male pigs (German Landrace $\times$ Pietrain, age 7 months) with indwelling vein catheters were used in total. At least 14 days before the beginning of blood sampling, Vena cephalica cannulation was performed under generalized anesthesia. Surgery was performed as previously described (44) with few modifications (45). The barrows were housed individually in pens $\left(5.4 \mathrm{~m}^{2}\right)$ with visual and tactile contact to their conspecifics. Pens were littered with dust-free wood shavings and cleaned every day after feeding. Light was on from 06:30 until 20:30. Pigs were fed hay ad libitum and concentrate $(1.5 \mathrm{~kg} / \mathrm{meal}, \mathrm{ME} 12 \mathrm{MJ} / \mathrm{kg})$ twice a day in the morning at 07:30 and in the afternoon at 15:00. To ensure blood sampling without disturbance of the animals, pigs were thoroughly habituated to human handling. Catheters were rinsed with heparinized saline (115 IU/mL; B. Braun Melsungen AG, Melsungen, Germany) every day during feeding in the morning. For blood collection via the catheters, $5 \mathrm{~mL}$ of blood was drawn and discarded before $10 \mathrm{~mL}$ blood per animal was collected into lithium heparin tubes (Sarstedt, Nümbrecht, Germany). 
Separation of peripheral blood mononuclear cells (PBMCs) from whole blood was performed with Leucosep ${ }^{\mathrm{TM}}$ Centrifuge Tubes (Greiner Bio-One, Frickenhausen, Germany) using Biocoll with a density of $1.077 \mathrm{~g} / \mathrm{mL}$ (Biochrom, Berlin, Germany) as previously described (6). In brief, PBMCs were separated by a density gradient, and after two washing steps; cells were suspended in RPMI 1640 supplemented with 10\% fetal calf serum (FCS) and $50 \mu \mathrm{g} / \mathrm{mL}$ gentamycin (all Biochrom). Afterward, cell concentration was determined with a Z2 Coulter Counter (Beckman Coulter, Krefeld, Germany).

\section{Preparation of Bacterial Supernatants}

To acquire supernatants from bacteria grown in vitro in presence and absence of $0.1 \mathrm{mM}$ ADR, $0.1 \mathrm{mM} \mathrm{NA}$, or $0.02 \mathrm{mM}$ AC (Sigma-Aldrich, Taufkirchen, Germany), S. enterica serovar Typhimurium Zoosaloral his ${ }^{-155} / \mathrm{ade}^{-4}$ (S. Typhimurium; DSM-No: 11320), auxotroph for histidine and adenine was chosen. S. Typhimurium was first allowed to grow on LB agar overnight at $37^{\circ} \mathrm{C}[1 \%(\mathrm{wt} / \mathrm{vol})$ tryptone, $0.5 \%$ (wt/vol) yeast extract, $1 \%$ (wt/vol) $\mathrm{NaCl}$, and $1.5 \%$ (wt/vol) bacto agar]. A single colony was used to inoculate $25 \mathrm{~mL}$ of $\mathrm{LB}$ medium [1\% (wt/vol) tryptone, $0.5 \%$ (wt/vol) yeast extract, $1 \%$ (wt/vol) $\mathrm{NaCl}$ ]. After incubation overnight at $37^{\circ} \mathrm{C}$ and $180 \mathrm{rpm}$ shaking (Infors HT Ecotron), S. Typhimurium cells were harvested by centrifugation ( $3 \mathrm{~min}, 10,000 \times g$ ), washed, and resuspended in heat-treated serum-SAPI cultivation medium (29) to obtain an optical cell density at $600 \mathrm{~nm}$ of 2 (Diode Array HP 8462A, Hewlett Packard, Palo Alto, CA, United States). Heat-treated serumSAPI cultivation medium contains SAPI solution $[6.25 \mathrm{mM}$ $\mathrm{NH}_{4} \mathrm{NO}_{3}, 1.84 \mathrm{mM} \mathrm{KH}_{2} \mathrm{PO}_{4}, 3.35 \mathrm{mM} \mathrm{KCl}$, autoclaved; $1.01 \mathrm{mM}$ $\mathrm{MgSO}_{4}, 2.77 \mathrm{mM}$ glucose, $10 \mathrm{mM}$ HEPES pH 7.5 sterile filtered $(0.22 \mu \mathrm{m})], 30 \%$ (vol/vol) FCS (Sigma-Aldrich), which was heat inactivated at $55^{\circ} \mathrm{C}$ for 20 min prior to use and supplementation of $0.12 \mathrm{mM}$ adenine monohydrochloride and $0.13 \mathrm{mM} \mathrm{L}$ histidine. Serum-SAPI was used as it is the medium of choice for analysis of CA effects on bacteria $(15,29,30)$. Cultivation medium was inoculated with the cell suspension to obtain an $\mathrm{OD}_{600}$ of 0.01 . To triplicates of $20 \mathrm{~mL}$ inoculated serum-SAPI either $10^{-4} \mathrm{M} \mathrm{ADR}, 10^{-4} \mathrm{M} \mathrm{NA}$, or $2 \times 10^{-5} \mathrm{M}$ AC (SigmaAldrich), or no further compound was added and incubated at $37^{\circ} \mathrm{C}$ and shaking (180 rpm). As control, cultivation medium without bacterial cells and without CAs or AC was also incubated under the same conditions. After $8 \mathrm{~h}$ of growth, when cells were in the exponential growth phase, cells were harvested by centrifugation (15 $\mathrm{min}, 7,000 \mathrm{rpm})$ and the supernatant was sterile filtered $(0.22 \mu \mathrm{m})$, frozen in liquid nitrogen, and stored at $-80^{\circ} \mathrm{C}$. Cells were harvested for collection of supernatants at OD600 $=0.34$ (no addition), 0.47 (ADR), 0.49 (NA), and 0.36 (AC).

\section{Determination of CA Contents in Bacterial Supernatants via High-Performance Liquid Chromatography}

High-performance liquid chromatography (HPLC) with electrochemical detection was conducted to determine the concentration of CAs in bacterial supernatants grown in the presence of NA or ADR. The HPLC system (ISO-3100BM, Thermo Fisher Scientific) was connected to an electrochemical detector [Coulochem III, conditioning cell (model 50210A), analytical cell (model 5011A), Thermo Fisher Scientific]. The potentials of the cells were set at 300,50 , and $-250 \mathrm{mV}$. The system was equipped with the column Reprosil Pur 120 C18-AQ $(4.6 \times 75 \mathrm{~mm})$ (A. Maisch, Ammerbuch, Germany). Cat-APhase II was used as the mobile phase, with a flow rate of $1.1 \mathrm{~mL} / \mathrm{min}$. The sample preparation with alumina extraction were adapted from the method first described by Anton and Sayre (46). Bacterial supernatants were diluted (1:10,000 and $1: 20,000)$ to be in the range of the applied calibration curve. In brief, $1 \mathrm{~mL}$ of sample and $500 \mathrm{pg}$ of an internal standard (dihydroxybenzylamine; Thermo Fisher Scientific, Darmstadt, Germany) were added to extraction tubes containing $20 \mathrm{mg}$ aluminum oxide previously activated with $600 \mu \mathrm{L} 2 \mathrm{M}$ Tris/EDTA buffer ( $\mathrm{pH}$ 8.7). Samples were thoroughly mixed in an overhead shaker for $10 \mathrm{~min}$ and centrifuged at $1,000 \times g$ for $1 \mathrm{~min}\left(4^{\circ} \mathrm{C}\right)$. Samples were washed three times with $1 \mathrm{~mL}$ of $16.5 \mathrm{mM}$ Tris/EDTA buffer ( $\mathrm{pH} 8.1$ ), followed by centrifugation. The CAs were eluted by addition of $120 \mu \mathrm{L}$ eluting solution (Recipe, Munich, Germany), short mixing, and centrifugation at $1,000 \times g$ for $1 \mathrm{~min}\left(4^{\circ} \mathrm{C}\right)$. Aliquots of $50 \mu \mathrm{L}$ were injected into the HPLC system. The internal standard method using peak areas was applied to evaluate the concentration of the samples.

\section{Lymphocyte Proliferation Assay}

For investigation of lymphocyte proliferative capacity, a mitogen-induced lymphocyte proliferation assay was performed as previously described (47). In short, PBMCs were seeded into 96-well round-bottom cell culture plates (Neolab, Heidelberg, Germany) with $1.5 \times 10^{5}$ cells/well and either stimulated with $5 \mu \mathrm{g} / \mathrm{mL}$ concanavalin A (ConA) or $5 \mu \mathrm{g} / \mathrm{mL}$ pokeweed mitogen (PWM) (both Sigma-Aldrich) or left without stimulation. Subsequently, supernatants from the differently treated $S$. Typhimurium cultures were added in concentrations of either 5,10 , or $15 \%$ of the total cell culture volume. To guarantee similar growth conditions throughout the wells, pure serum-SAPI was applied to control wells as well as for volume compensation, resulting in $15 \%$ serum-SAPI-based additive in every well. Each treatment was done in triplicates. Cells were incubated at $39^{\circ} \mathrm{C}$, and $5 \% \mathrm{CO}_{2}$ for $48 \mathrm{~h}$ before $0.25 \mu \mathrm{Ci}{ }^{3} \mathrm{H}$-thymidine/well (PerkinElmer, Rodgau, Germany) was added, followed by a further incubation for $24 \mathrm{~h}$. PBMCs were harvested using glass fiber filters (Sigma-Aldrich), and the incorporated radioactivity was measured by a liquid scintillation analyzer (PerkinElmer). For each treatment, the mean of counts per minute $(\mathrm{cpm})$ was calculated, and the mean cpm of the unstimulated control was subtracted to gain $\Delta \mathrm{cpm}$.

HPLC analysis of the Salmonella supernatants showed that substantial amounts of CAs were still present in CA-treated cultures. We thus performed an additional experiment to ensure that probable bacterial effects were not in fact caused by CAs or by mere synergistic effects of bacterial products and CAs. Therefore, previously frozen PBMCs of three animals were thawed and seeded with $1.5 \times 10^{5}$ cells/well in 96-well round-bottom cell 
culture plates in RPMI 1640 supplemented with 10\% FCS and $50 \mu \mathrm{g} / \mathrm{mL}$ gentamycin. Cells were incubated at $39^{\circ} \mathrm{C}$ and $5 \%$ $\mathrm{CO}_{2}$ as described for the first experiment after adding one of the following treatments: PBMCs were either left unstimulated after addition of $15 \%$ serum-SAPI medium or stimulated with $5 \mu \mathrm{g} / \mathrm{mL}$ ConA. Stimulated cells were supplemented with one of the following additives: $15 \%$ serum-SAPI alone, $15 \%$ serumSAPI and $10^{-5} \mathrm{M}$ NA, $15 \%$ serum-SAPI and $10^{-5} \mathrm{M}$ ADR, $15 \%$ supernatants from $S$. Typhimurium grown without hormone, $15 \%$ supernatants from $S$. Typhimurium grown in the presence of $10^{-4} \mathrm{M} \mathrm{NA}$ or $10^{-4} \mathrm{M}$ ADR, or $15 \%$ supernatants from $S$. Typhimurium grown without hormone with retrospective addition of $10^{-5} \mathrm{M}$ NA or $10^{-5} \mathrm{M}$ ADR.

In a third experiment, lymphocyte proliferation was assessed again as described previously but with addition of AC (SigmaAldrich). As the effective concentration (and the amount of presumed ADR oxidation in Salmonella cultures) was unknown, we investigated a wide range of concentrations $\left(10^{-10}\right.$ to $10^{-5}$ $\mathrm{M})$. After addition of $\mathrm{AC}$ and stimulation with $5 \mu \mathrm{g} / \mathrm{mL}$ ConA or $5 \mu \mathrm{g} / \mathrm{mL}$ PWM, cells were incubated, and proliferation was determined as described above.

\section{Statistical Analysis}

For statistical analysis, we used the software SAS, version 9.4 (SAS institute Inc., Cary, NC, United States), applying the MIXED procedure. Degrees of freedom were determined with the Kenward-Roger method (48); normal distribution and variance homogeneity were confirmed visually by normal probability plots and plots of residuals versus fitted values (49). For estimation of variance components, we used the restricted maximum likelihood method. The models included the factors "treatment" and "trial," as well as their interaction as fixed effects and "sampling day" and "sampling day $\times$ treatment" as random effects. To take into account the individual level of the pigs, "animal" was included as a repeated effect. If data were not normally distributed, logarithmic or square root transformation was performed to attain normality. The results are presented as least square (ls)-means + standard error of the mean (SEM). Statistically significant differences were determined by Fisher's least significant difference test. Significance limits were set as follows: ${ }^{*} p<0.05,{ }^{* *} p<0.01$, ${ }^{* * *} p<0.001$, and ${ }^{t} p<0.1$ (tendency).

\section{RESULTS}

\section{Supernatants From CA-Treated S. Typhimurium Cultures Inhibit Lymphocyte Proliferation}

We first evaluated the effects of supernatants from $S$. Typhimurium cultures on lymphocyte proliferation. Compared to the media control, the addition of supernatants from hormone-free Salmonella cultures enhanced ConAinduced lymphocyte proliferation (Figure 1A). In comparison to supernatants from hormone-free Salmonella cultures, lymphocyte proliferation was reduced significantly if $10 \%$ or $15 \%$

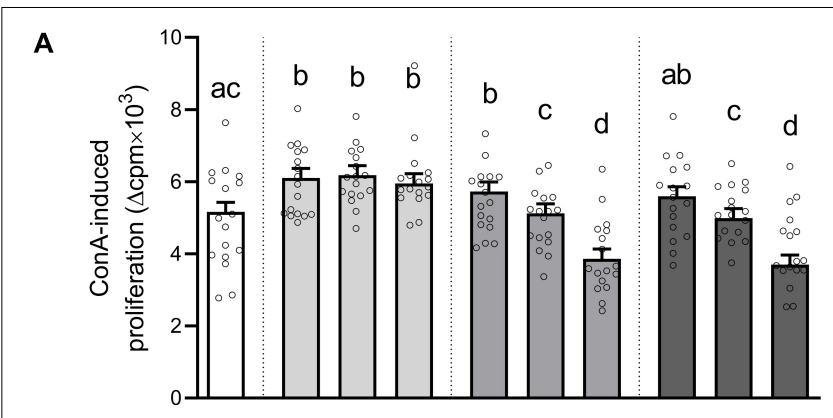

B

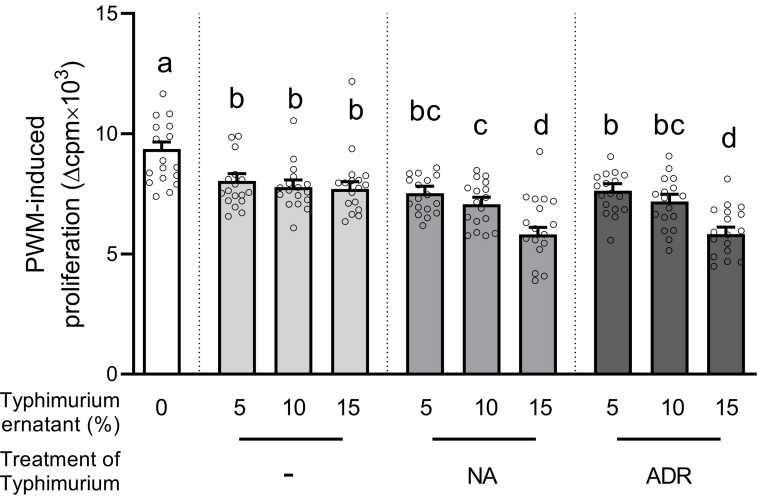

FIGURE 1 | Lymphocyte proliferation after stimulation with either (A) concanavalin A (ConA) or (B) pokeweed mitogen (PWM), as well as addition of either serum-SAPI medium (white) or supernatants from Salmonella Typhimurium cultures grown for $8 \mathrm{~h}$ at $37^{\circ} \mathrm{C}$ without hormones (light gray) or in the presence of $10^{-4} \mathrm{M}$ noradrenaline (NA; medium gray) or $10^{-4} \mathrm{M}$ adrenaline (ADR; dark gray). Supernatants were added in concentrations of either $5 \%, 10 \%$, or $15 \%$ of the cell culture volume as indicated on the $x$ axis. Treatments that are statistically significant from each other are indicated by different letters on top of their bars, whereas bars that share a common letter do not differ significantly. Data are presented as Is-means + SEM (bars) and single values of each animal (circles), $n=16$.

of supernatants from Salmonella grown in the presence of ADR or NA were added and already tended to be lower $(p=0.053)$ if $5 \%$ of supernatants from Salmonella grown in the presence of ADR were added. In PWM-stimulated PBMCs, already the addition of supernatants from hormone-free Salmonella cultures reduced proliferation compared to the media control (Figure 1B). But similar to ConA-stimulated cells, addition of $10 \%$ or $15 \%$ of supernatants from Salmonella grown in the presence of NA further reduced proliferation significantly. The addition of supernatants from Salmonella grown in the presence of ADR caused a less pronounced suppression of PWM-stimulated cells with a significant effect if $15 \%$ and a tendency $(p=0.058)$ if $10 \%$ were added.

\section{Suppression of Lymphocyte Function Is Not Due to CA Action}

Because CAs themselves are well-described to modulate immune cell functionality, we determined whether CAs were still present in $S$. Typhimurium cultures incubated for $8 \mathrm{~h}$ in the presence of either NA or ADR by HPLC analysis. Thereby, an ADR 
A

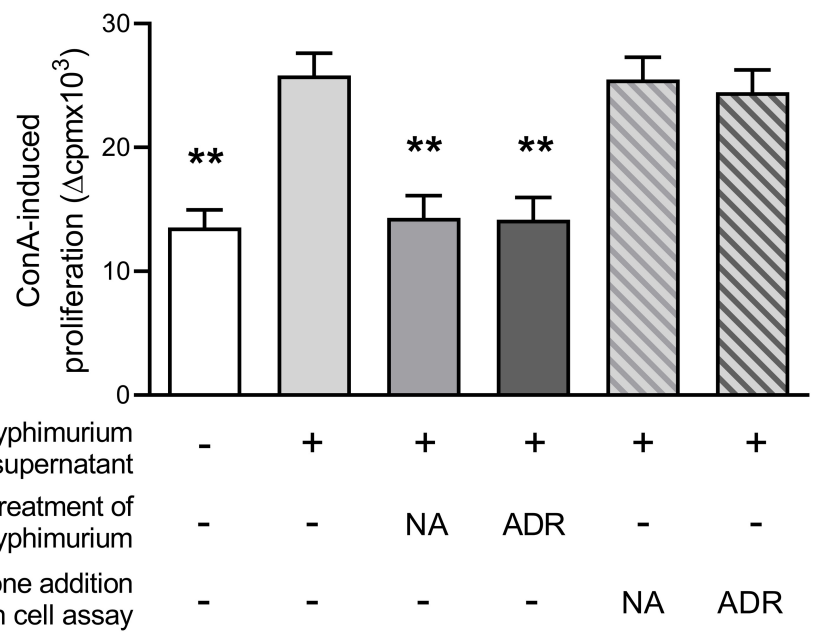

B

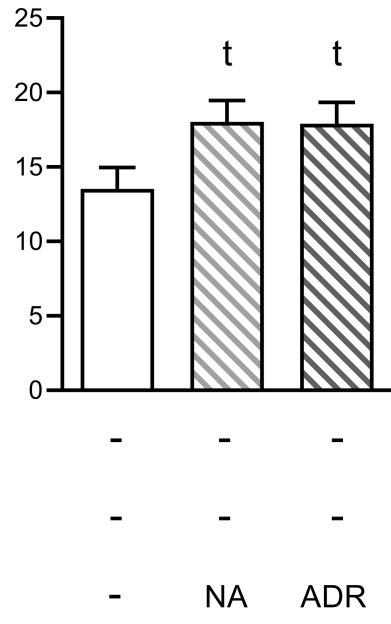

FIGURE 2 | Lymphocyte proliferation after stimulation with $5 \mu \mathrm{g} / \mathrm{mL}$ concanavalin A and upon addition of 15\% serum-SAPI (white), 15\% supernatants from Salmonella Typhimurium cultures grown without hormones (light gray) or grown with either $10^{-4} \mathrm{M}$ noradrenaline (NA; middle gray) or $10^{-4} \mathrm{M}$ adrenaline (ADR; dark gray) for $8 \mathrm{~h}$ at $37^{\circ} \mathrm{C}$, or addition of $15 \%$ supernatants from S. Typhimurium cultures grown without hormones simultaneous to catecholamine addition [ $10^{-5} \mathrm{M}$ NA (light gray hatched in middle gray) or $10^{-5} \mathrm{M} \mathrm{ADR}$ (light gray hatched in dark gray)] (A); or upon addition of $15 \%$ serum-SAPI without further additives (white) or additional supplementation with $10^{-5} \mathrm{M} \mathrm{NA}$ (white hatched in middle gray) or $10^{-5} \mathrm{M}$ ADR (white hatched in dark gray) (B). Data are presented as Is-means + SEM, $n=3$. Asterisks and $t$ in superscript indicate significant differences and tendencies compared to supernatants from hormone-free Salmonella culture (A) or the hormone-free control $\mathbf{( B )}$, respectively.

concentration of $19.67 \mu \mathrm{g} / \mathrm{mL}\left(1.07 \times 10^{-4} \mathrm{M}\right)$ was found, representing the same level as applied at the start of incubation $\left(1 \times 10^{-4} \mathrm{M}\right)$. NA showed a slight decrease compared to the initial concentration of $1 \times 10^{-4} \mathrm{M}$, but was still present in the supernatants at a concentration of $8.08 \mu \mathrm{g} / \mathrm{mL}\left(4.8 \times 10^{-5} \mathrm{M}\right)$. Thus, to verify that probable bacterial effects were not "ordinary" immunomodulating effects of CAs or caused by mere synergistic effects of bacterial products and CAs, we tested the effects of simultaneous addition of supernatant from $S$. Typhimurium grown without hormones and either NA or ADR in the same range as found within the culture supernatants tested in the initial experiment (cf. Figure 1).

As seen in Figure 2A, ConA-induced lymphocyte proliferation was significantly lower if supernatants from Salmonella grown in the presence of NA or ADR were added compared to supernatants from hormone-free Salmonella culture. Thus, the results presented above (cf. Figure 1) could be confirmed. Notably, in contrast to this effect, no suppression occurred on ConA-induced lymphocyte proliferation if supernatants from hormone-free Salmonella cultures were added simultaneously with ADR or NA (Figure 2A). Opposite to the effect of supernatants from Salmonella grown in the presence of NA or ADR, proliferation was slightly increased if cells were treated with NA $(p=0.073)$ or $\operatorname{ADR}(p=0.068)$ alone (Figure 2B).

\section{The ADR Oxidation Product AC Is Not the Active Inhibitory Agent in Supernatants From CA-Treated Salmonella Cultures}

To assess whether the oxidation of CAs by Salmonella might cause the observed suppressive effect of supernatants from CA-treated bacterial cultures, we performed the lymphocyte proliferation assay under the same conditions as in the first experiment (cf. Figure 1) but added AC instead of bacterial supernatants (Figures 3A,B). If PBMCs were stimulated with ConA, all tested concentrations led to an enhancement of proliferation compared to the AC-free control (Figure 3A), whereas no effect was observed upon stimulation with PWM (Figure 3B).

Because AC can also have a direct effect on bacteria, like in $V$. cholerae (41), we assumed that its effect on PBMCs might possibly be mediated indirectly, by modulating the behavior of $S$. Typhimurium upon sensing. In addition to the treatment of Salmonella cultures with NA or ADR, we thus also cultured $S$. Typhimurium with $2 \times 10^{-5} \mathrm{M}$ AC for $8 \mathrm{~h}$ at $37^{\circ} \mathrm{C}$ before centrifugation and microfiltration. If supernatant from these cultures was added to ConA-stimulated PBMCs, proliferation was enhanced compared to the serum-SAPIcontrol but not significantly different from the proliferation upon addition of supernatants from hormone-free Salmonella cultures (Figure 3C). If PWM was used, proliferation was lower than upon serum-SAPI addition and on the same level as with the supernatant from hormone-free Salmonella cultures (Figure 3D).

\section{DISCUSSION}

The results of the present study indicate a close host-pathogen cross-talk in situations with elevated stress hormone levels in pigs. Based on pioneering work demonstrating the ability of many bacteria to increase pathogenicity in response to CAs (23, 50 ), we here show that interkingdom signaling also works the other way. Our data indicate that there is a direct action of CA-treated bacteria on host immune cells. Lymphocytes treated 


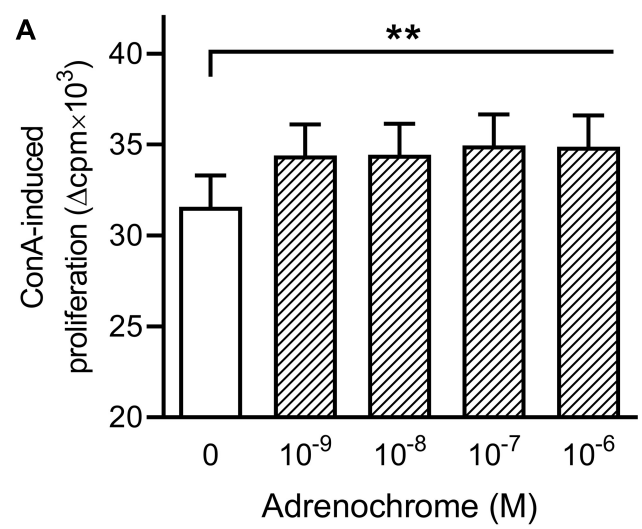

C

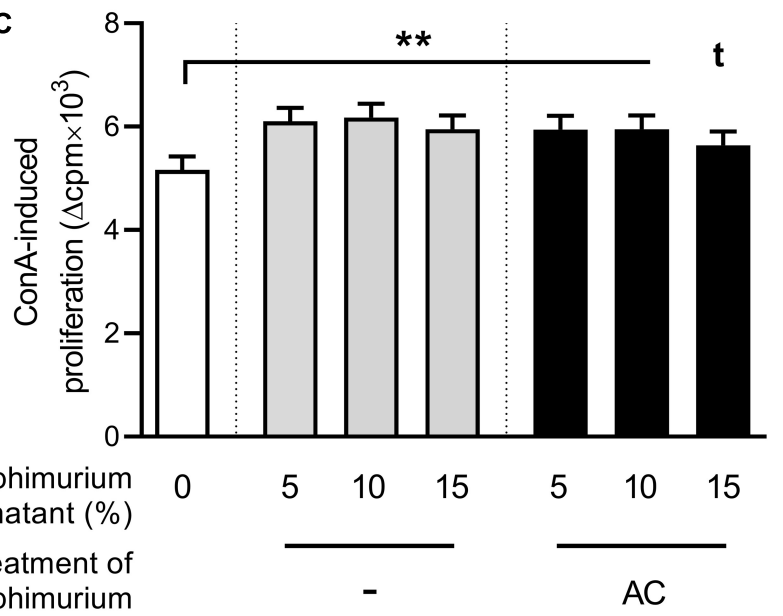

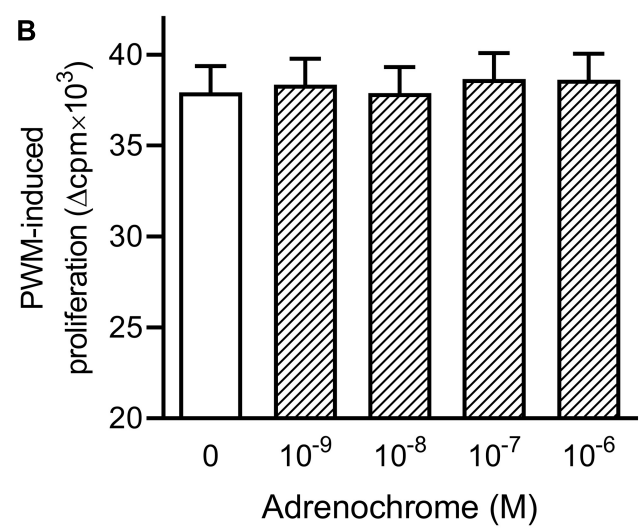

D $127 \quad \vdots \quad * * *$

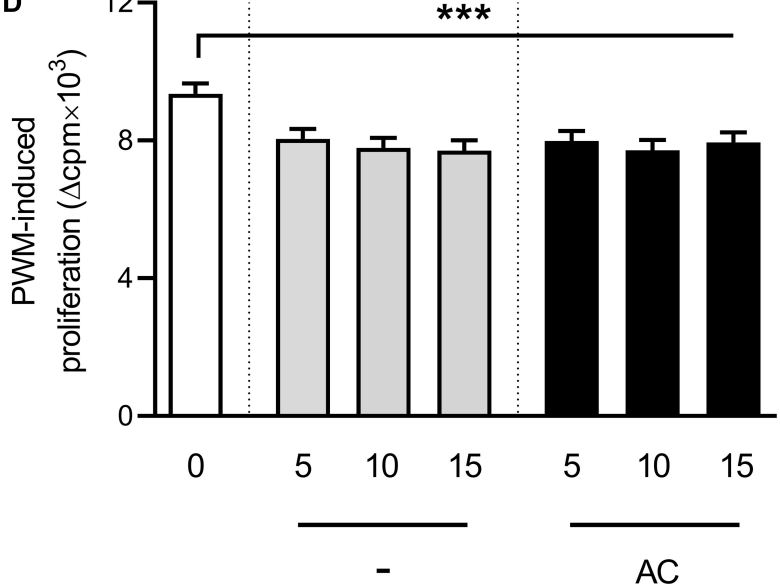

FIGURE 3 | Lymphocyte proliferation upon addition of adrenochrome (A,B) or serum-SAPI medium or supernatants from Salmonella Typhimurium cultures grown without additive or in the presence of $2 \times 10^{-5} \mathrm{M}$ adrenochrome for $8 \mathrm{~h}$ at $37^{\circ} \mathrm{C}$ (C,D), and stimulation with either $5 \mu \mathrm{g} / \mathrm{mL} \mathrm{concanavalin} \mathrm{A}[\mathrm{ConA}$; (A,C)] or $5 \mu \mathrm{g} / \mathrm{mL}$ pokeweed mitogen [PWM; (B,D)]. Significant differences are marked by asterisks, tendencies are indicated by a $t$ in superscript. Data are presented as Is-means + SEM, $n=19$ (A,B), $n=16$ (C,D).

with cell-free supernatants from $S$. Typhimurium grown in the presence of ADR or NA showed a decreased proliferation, which is probably not the only hampered immune function. Future studies should investigate further important immune functions such as the production of pro-inflammatory cytokines, which are also involved in Salmonella control (51).

We demonstrate that the inhibition of lymphocyte proliferation does not simply reflect an immunomodulating effect of CAs, as retrospective addition of ADR or NA in combination with supernatant of non-treated $S$. Typhimurium did not inhibit mitogen-induced proliferation of porcine immune cells. This is also supported by our previous study, showing that under the same cell culture conditions, the sole addition of ADR or NA led to an increased lymphocyte proliferation instead of its reduction (6). This implies that the proposed immunosuppressive substance produced by CA-treated $S$. Typhimurium must be very potent if it even diminishes the enhancing effect of the CAs that were still present in the supernatants.
To the best of our knowledge, this is the first study to report that bacteria grown in the presence of stress hormones alter their growth environment-probably by producing immunomodulating substances-in a way that host immune response is impaired.

Based on own previous studies, AC was a promising candidate for the observed immunosuppression by $S$. Typhimurium. These experiments demonstrated that AC was formed during bacterial culture of $V$. cholerae $(29,41)$ upon ADR addition, and AC treatment of the human monocytic cell line THP-1 caused a hampered TNF- $\alpha$ production (41). Also, it is already known that AC can bind to ARs (39), which are present on all immune cells (4). We thus investigated whether this oxidation product of ADR may be responsible for the observed effects on porcine primary immune cells. However, AC either added directly to porcine lymphocytes or added to $S$. Typhimurium cultures did not decrease porcine lymphocyte functionality but instead had no effect or even increased it. Based on these results, it can be ruled out that $\mathrm{AC}$ is the immunomodulating substance responsible 
for the observed inhibition. Thus, $S$. Typhimurium must have produced different signaling molecule(s). At this point, it can only be speculated as to what substance might be responsible for the findings by comparing the demonstrated effects with those attributed to already identified molecules that are produced by $S$. Typhimurium or other bacteria.

It was shown that NA triggers the release of autoinducers (AIs) in many Gram-negative bacteria including Salmonella (16). This group of quorum-sensing molecules not only enhances the growth and virulence of the bacteria themselves but may also influence the host immune system. The most prominently mentioned and potentially immunomodulatory AI in the literature is AI-3, which is also produced by $S$. Typhimurium $(36,52)$. Although the exact structure still remains unknown, it has an aminated aromatic compound and seems to have a high similarity to CAs because it can be blocked by $\alpha$ - and $\beta$ adrenergic antagonists (53-55), and both NA and AI-3 can bind to QseC (27). It is thus likely that AI-3 can bind to mammalian ARs. However, we have previously shown by in vitro culture with CAs that AR binding leads to increased proliferation of porcine PBMCs, contrary to the effects of supernatants from ADR- or NA-treated Salmonella presented here (6). Also, an $\alpha$-adrenergic action of AI-3 is unlikely as binding to these receptors generally causes an enhanced immune functionality $(4,9)$. Nevertheless, it cannot be precluded at this point that AI-3 might specifically bind to $\beta_{2}$-ARs in mammalian immune cells, which are mostly immunosuppressive (56).

There is a second important AI molecule produced by $S$. Typhimurium in the exponential growth phase, named AI2 (57). It plays a role in invasion and intracellular survival in macrophages $(58,59)$, but indications for a direct modulation of host immune cells have not been found so far. Whether this is a candidate for immunosuppression by Salmonella in a stressed host may be subject of future studies.

Another interesting class of bacterial hormone-like molecules is the lipophilic acyl homoserine lactones (AHLs). They are chemically analogous to eukaryotic lipid hormones and can either impair or exacerbate immune functions, depending on their concentration. It has even been shown that they have the ability to inhibit lymphocyte proliferation and TNF- $\alpha$ production in macrophages and $\mathrm{T}_{H}$ cells $(32,60,61)$. Although this very much resembles the findings of the present study, an AHL production was so far not described in Salmonella species (62).

Also, it was shown that $S$. Typhimurium can deacylate the lipid A portion of their lipopolysaccharide, which results in a lower activation of Toll-like receptor 4 on antigen-presenting cells. As a consequence, the immune-activating intracellular nuclear factor $\kappa \mathrm{B}$ signaling, as well as the release of pro-inflammatory cytokines, is hampered (63). It is conceivable that the effects observed in the present study may at least partly be caused by an activation of this mechanism upon CA sensing of the bacteria.

Conclusively, this study added further novel clues to explain the increased susceptibility of a stressed host to infection. It has been shown earlier that stress has a negative impact on Salmonella recrudescence in pigs by increasing intracellular Salmonella proliferation in macrophages (64). A direct effect on invasiveness and intracellular survival rate of $S$. Typhimurium by binding of NA to the histidine kinase QseC was demonstrated in another study in mice (65). S. Typhimurium infection in calves was also aggravated by an increase of bacterial proliferation by NA, probably through acting as an iron donor for the bacteria (66). The present work shows for the first time that bacteria grown under the influence of NA or ADR are even able to hamper mammalian lymphocyte functionality. Thus, valuable information is added to the phenomenon of increased Salmonella susceptibility of stressed pigs. Pigs represent an important meatproducing agricultural species and are relevant carriers of the widely distributed zoonotic agent $S$. Typhimurium (67). At the same time, pigs are an excellent model for human salmonellosis because porcine nutritional physiology and gut anatomy as well as the immune system are very similar to that of humans (68-71). Upon this basic study, it is thus possible to make presumptions about effects of stress on the risk of salmonellosis in humans, i.e., increased risk of infection due to immunosuppression by CAprimed bacteria, while at the same time gaining knowledge about porcine immunology that may have impacts on pig husbandry and food hygiene at the slaughterhouse.

\section{DATA AVAILABILITY STATEMENT}

The raw data supporting the conclusion of this article will be made available by the authors, without undue reservation.

\section{ETHICS STATEMENT}

The animal study was reviewed and approved by the Regierungspräsidium Stuttgart.

\section{AUTHOR CONTRIBUTIONS}

VS and JS conceived and designed the study. VS, JS, SS, CT, and LR designed the experiments. CT produced bacterial supernatants. BP conducted the CA analyses. LR performed and SSS supervised the immunological experiments. LR analyzed and interpreted the data, and wrote the original draft of the manuscript. VS, JS, SS, CT, and BP contributed to the manuscript preparation. All authors have read and agreed to the published version of the manuscript.

\section{FUNDING}

This research was funded by the German Research Foundation (DFG), grant number STE 633/10-1.

\section{ACKNOWLEDGMENTS}

The authors thank Ulrike Weiler, Larissa Engert, Tanja Hofmann, and Philipp Marro for surgical assistance, Petra Veit and Susanne Rautenberg for assistance in the laboratory, and William Dunne, Mohammed Mecellem, Manuela Ganser, and Claudia Fischinger for excellent animal care. They also thank Charlotte Heyer for preliminary work on this project and Filippo Capezzone for statistical advice. 


\section{REFERENCES}

1. Gordan R, Gwathmey JK, Xie L-H. Autonomic and endocrine control of cardiovascular function. World J Cardiol. (2015) 7:204-14. doi: 10.4330/wjc. v7.i4.204

2. Kyrou I, Tsigos C. Stress hormones: physiological stress and regulation of metabolism. Curr Opin Pharmacol. (2009) 9:787-93. doi: 10.1016/j.coph.2009. 08.007

3. Antonelli A, Torchio R, Bertolaccini L, Terzi A, Rolfo F, Agostoni P, et al. Contribution of $\beta$-adrenergic receptors to exercise-induced bronchodilatation in healthy humans. Respir Physiol Neurobiol. (2012) 184:55-9. doi: 10.1016/j. resp.2012.07.007

4. Kohm A, Sanders VM. Norepinephrine: a messenger from the brain to the immune system. Trends Immunol. (2000) 21:539-42.

5. Elenkov IJ, Wilder RL, Chrousos GP, Vizi ES. The sympathetic nerv-an integrative interface between two supersystems: the brain and the immune system. Pharmacol Rev. (2000) 52:595-638.

6. Reiske L, Schmucker S, Steuber J, Stefanski V. Glucocorticoids and catecholamines affect in vitro functionality of porcine blood immune cells. Animals. (2019) 9:545. doi: 10.3390/ani9080545

7. Kouassi E, Li YS, Boukhris W, Millet I, Revillard JP. Opposite effects of the catecholamines dopamine and norepinephrine on murine polyclonal B-cell activation. Immunopharmacology. (1988) 16:125-37.

8. Torres KC, Antonelli LR, Souza AL, Teixeira MM, Dutra WO, Gollob KJ. Norepinephrine, dopamine and dexamethasone modulate discrete leukocyte subpopulations and cytokine profiles from human PBMC. J Neuroimmunol. (2005) 166:144-57. doi: 10.1016/j.jneuroim.2005.06.006

9. Hadden JW, Hadden EM, Middleton E. Lymphocyte blast transformation. I. Demonstration of adrenergic receptors in human peripheral lymphocytes. Cell Immunol. (1970) 1:583-95.

10. Felten SY, Olschowka J. Noradrenergic sympathetic innervation of the spleen: II. Tyrosine hydroxylase (TH)-positive nerve terminals form synapticlike contacts on lymphocytes in the splenic white pulp. J Neurosci Res. (1987) 18:37-48. doi: 10.1002/jnr.490180108

11. Bergquist J, Tarkowski A, Ewing A, Ekman R. Catecholaminergic suppression of immunocompetent cells. Immunol Today. (1998) 19:562-7.

12. Althen TG, Ono K, Topel DG. Effect of stress susceptibility or stunning method on catecholamine levels in swine. J Anim Sci. (1977) 44:985-9. doi: $10.2527 /$ jas1977.446985x

13. Bacou E, Haurogné K, Mignot G, Allard M, de Beaurepaire L, Marchand J, et al. Acute social stress-induced immunomodulation in pigs high and low responders to ACTH. Physiol Behav. (2017) 169:1-8. doi: 10.1016/j.physbeh. 2016.11.012

14. Asano Y, Hiramoto T, Nishino R, Aiba Y, Kimura T, Yoshihara K, et al. Critical role of gut microbiota in the production of biologically active, free catecholamines in the gut lumen of mice. Am J Physiol Gastrointest Liver Physiol. (2012) 303:G1288-95. doi: 10.1152/ajpgi.00341.2012

15. Lyte M, Ernst S. Catecholamine induced growth of gram negative bacteria. Life Sciences. (1992) 50:203-12.

16. Freestone PP, Haigh RD, Williams PH, Lyte M. Stimulation of bacterial growth by heat-stable, norepinephrine-induced autoinducers. FEMS Microbiol Lett. (1999) 172:53-60. doi: 10.1111/j.1574-6968.1999.tb13449.x

17. Roberts A, Matthews JB, Socransky SS, Freestone PP, Williams PH, Chapple IL. Stress and the periodontal diseases: effects of catecholamines on the growth of periodontal bacteria in vitro. Oral Microbiol Immunol. (2002) 17:296-303. doi: 10.1034/j.1399-302X.2002.170506.x

18. Aneman A, Eisenhofer G, Olbe L, Dalenbäck J, Nitescu P, Fändriks L, et al. Sympathetic discharge to mesenteric organs and the liver. Evidence for substantial mesenteric organ norepinephrine spillover. J Clin Invest. (1996) 97:1640-6.

19. Kong HH. Skin microbiome: genomics-based insights into the diversity and role of skin microbes. Trends Mol Med. (2011) 17:320-8. doi: 10.1016/j. molmed.2011.01.013

20. Dewhirst FE, Chen T, Izard J, Paster BJ, Tanner AC, Yu W-H, et al. The human oral microbiome. J Bacteriol. (2010) 192:5002-17. doi: 10.1128/JB.00542-10

21. Ley RE, Peterson DA, Gordon JI. Ecological and evolutionary forces shaping microbial diversity in the human intestine. Cell. (2006) 124:837-48. doi: 10. 1016/j.cell.2006.02.017
22. Lyte M, Erickson AK, Arulanandam BP, Frank CD, Crawford MA, Francis DH. Norepinephrine-induced expression of the K99 pilus adhesin of enterotoxigenic Escherichia coli. Biochem Biophys Res Commun. (1997) 232:682-6. doi: 10.1006/bbrc.1997.6356

23. Freestone PP, Sandrini SM, Haigh RD, Lyte M. Microbial endocrinology: How stress influences susceptibility to infection. Trends Microbiol. (2008) 16:55-64. doi: 10.1016/j.tim.2007.11.005

24. Miethke M, Skerra A. Neutrophil gelatinase-associated lipocalin expresses antimicrobial activity by interfering with L-norepinephrine-mediated bacterial iron acquisition. Antimicrob Agents Chemother. (2010) 54:1580-9. doi: 10.1128/AAC.01158-09

25. Schaible UE, Kaufmann SH. Iron and microbial infection. Nat Rev Microbiol. (2004) 2:946-53. doi: 10.1038/nrmicro1046

26. Bearson BL, Bearson SM. The role of the QseC quorum-sensing sensor kinase in colonization and norepinephrine-enhanced motility of Salmonella enterica serovar Typhimurium. Microb Pathog. (2008) 44:271-8. doi: 10.1016/ j.micpath.2007.10.001

27. Clarke MB, Hughes DT, Zhu C, Boedeker EC, Sperandio V. The QseC sensor kinase: a bacterial adrenergic receptor. Proc Natl Acad Sci USA. (2006) 103:10420-5. doi: 10.1073/pnas.0604343103

28. Hegde M, Wood TK, Jayaraman A. The neuroendocrine hormone norepinephrine increases Pseudomonas aeruginosa PA14 virulence through the las quorum-sensing pathway. Appl Microbiol Biotechnol. (2009) 84:763-76. doi: 10.1007/s00253-009-2045-1

29. Halang P, Toulouse C, Geißel B, Michel B, Flauger B, Müller M, et al. Response of Vibrio cholerae to the catecholamine hormones epinephrine and norepinephrine. J Bacteriol. (2015) 197:3769-78. doi: 10.1128/JB.00345-15

30. Freestone PP, Haigh RD, Lyte M. Specificity of catecholamine-induced growth in Escherichia coli O157:H7, Salmonella enterica and Yersinia enterocolitica. FEMS Microbiol Lett. (2007) 269:221-8. doi: 10.1111/j.1574-6968.2006. 00619.x

31. Waters CM, Bassler BL. Quorum sensing: cell-to-cell communication in bacteria. Annu Rev Cell Dev Biol. (2005) 21:319-46. doi: 10.1146/annurev. cellbio.21.012704.131001

32. Telford G, Wheeler D, Williams P, Tomkins PT, Appleby P, Sewell H, et al. The Pseudomonas aeruginosa quorum-sensing signal molecule N(3-Oxododecanoyl)-1-homoserine lactone has immunomodulatory activity. Infect Immun. (1998) 66:36-42.

33. Smith RS, Harris SG, Phipps R, Iglewski B. The Pseudomonas aeruginosa quorum-sensing molecule $\mathrm{N}$-(3-Oxododecanoyl)homoserine lactone contributes to virulence and induces inflammation In Vivo. $J$ Bacteriol. (2002) 184:1132-9. doi: 10.1128/jb.184.4.1132-1139.2002

34. Zimmermann S, Wagner C, Müller W, Brenner-Weiss G, Hug F, Prior B, et al. Induction of neutrophil chemotaxis by the quorum-sensing molecule N(3-Oxododecanoyl)-1-homoserine lactone. Infect Immun. (2006) 74:5687-92. doi: 10.1128/IAI.01940-05

35. Mota LJ, Cornelis GR. The bacterial injection kit: type III secretion systems. Ann Med. (2005) 37:234-49. doi: 10.1080/07853890510037329

36. Sperandio V, Torres AG, Jarvis B, Nataro JP, Kaper JB. Bacteria-host communication: the language of hormones. Proc Natl Acad Sci USA. (2003) 100:8951-6. doi: 10.1073/pnas.1537100100

37. Bors W, Michel C, Manfred S, Lengfelder E. The involvement of oxygen radicals during the autoxidation of adrenalin. Biochim Biophys Acta Gen Subj. (1978) 540:162-72. doi: 10.1016/0304-4165(78)90445-2

38. Alhasan R, Njus D. The epinephrine assay for superoxide: why dopamine does not work. Anal Biochem. (2008) 381:142-7. doi: 10.1016/j.ab.2008.06.030

39. Yates JC, Taam GM, Singal PK, Beamish RE, Dhalla NS. Protection against adrenochrome-induced myocardial damage by various pharmacological interventions. Br J Exp Pathol. (1980) 61:242-55.

40. Marino F, Cosentino M. Adrenergic modulation of immune cells: an update. Amino Acids. (2013) 45:55-71. doi: 10.1007/s00726-011-1186-6

41. Toulouse C, Schmucker S, Metesch K, Pfannstiel J, Michel B, Starke I, et al. Mechanism and impact of catecholamine conversion by Vibrio cholerae. Biochim Biophys Acta Bioenerg. (2019) 1860:478-87. doi: 10.1016/j.bbabio. 2019.04.003

42. Verbrugghe E, Boyen F, Gaastra W, Bekhuis L, Leyman B, van Parys A, et al. The complex interplay between stress and bacterial infections in animals. Vet Microbiol. (2012) 155:115-27. doi: 10.1016/j.vetmic.2011.09.012 
43. Verbrugghe E, Dhaenens M, Leyman B, Boyen F, Shearer N, van Parys A, et al. Host stress drives Salmonella recrudescence. Sci Rep. (2016) 6:20849. doi: 10.1038/srep20849

44. Kraetzl WD, Weiler U. Erfahrungen mit einem implantierbaren Kathetersystem zur frequenten und chronischen Blutentnahme bei Schafen in Gruppenhaltung und bei säugenden Sauen. Tierarztl Umsch. (1998) 53:567-74.

45. Engert LC, Weiler U, Pfaffinger B, Stefanski V, Schmucker SS. Diurnal rhythms in peripheral blood immune cell numbers of domestic pigs. Dev Comp Immunol. (2018) 79:11-20. doi: 10.1016/j.dci.2017.10.003

46. Anton AH, Sayre DF. A study of the factors affecting the aluminum oxidetrihydroxyindole procedure for the analysis of catecholamines. J Pharmacol Exp Ther. (1962) 138:360-75.

47. Schalk C, Pfaffinger B, Schmucker S, Weiler U, Stefanski V. Effects of repeated social mixing on behavior and blood immune cells of group-housed pregnant sows (Sus scrofa domestica). Livest Sci. (2018) 217:148-56. doi: 10.1016/j.livsci. 2018.09.020

48. Kenward MG, Roger JH. Small sample inference for fixed effects from restricted maximum likelihood. Biometrics. (1997) 53:983. doi: 10.2307/ 2533558

49. Faraway JJ. Extending the Linear Model With R: Generalized Linear, Mixed Effects and Nonparametric Regression Models. Boca Raton, FL: Chapman \& Hall/CRC (2006)

50. Lyte M, Vulchanova L, Brown DR. Stress at the intestinal surface: catecholamines and mucosa-bacteria interactions. Cell Tissue Res. (2011) 343:23-32. doi: 10.1007/s00441-010-1050-0

51. Dolowschiak T, Mueller AA, Pisan LJ, Feigelman R, Felmy B, Sellin ME, et al. IFN-gamma hinders recovery from mucosal inflammation during antibiotic therapy for Salmonella gut infection. Cell Host Microbe. (2016) 20:238-49. doi: 10.1016/j.chom.2016.06.008

52. Walters M, Sircili MP, Sperandio V. AI-3 synthesis is not dependent on luxS in Escherichia coli $\uparrow$. J Bacteriol. (2006) 188:5668-81. doi: 10.1128/JB.00 648-06

53. Clarke MB, Sperandio V. Events at the host-microbial interface of the gastrointestinal tract III. Cell-to-cell signaling among microbial flora, host, and pathogens: there is a whole lot of talking going on. Am J Physiol Gastrointest Liver Physiol. (2005) 288:G1105-9. doi: 10.1152/ajpgi.00572. 2004

54. Bearson BL, Bearson SM, Lee IS, Brunelle BW. The Salmonella enterica serovar Typhimurium QseB response regulator negatively regulates bacterial motility and swine colonization in the absence of the QseC sensor kinase. Microb Pathog. (2010) 48:214-9. doi: 10.1016/j.micpath.2010.03.005

55. Yang Q, Anh ND, Bossier P, Defoirdt T. Norepinephrine and dopamine increase motility, biofilm formation, and virulence of Vibrio harveyi. Front Microbiol. (2014) 5:584. doi: 10.3389/fmicb.2014.00584

56. Fan X, Wang Y. $\beta 2$ Adrenergic receptor on $\mathrm{T}$ lymphocytes and its clinical implications. Prog Nat Sci. (2009) 19:17-23. doi: 10.1016/j.pnsc.2008.10.001

57. Surette MG, Miller MB, Bassler BL. Quorum sensing in Escherichia coli, Salmonella typhimurium, and Vibrio harveyi: a new family of genes responsible for autoinducer production. Proc Natl Acad Sci USA. (1999) 96:1639-44. doi: 10.1073/pnas.96.4.1639

58. Choi J, Park J, Ryu S. Repressed Quorum sensing by overexpressing LsrR Hampers Salmonella evasion from oxidative killing within macrophages. $J$ Microbiol Biotechnol. (2010) 20:1624-9.

59. Choi J, Shin D, Kim M, Park J, Lim S, Ryu S. LsrR-mediated quorum sensing controls invasiveness of Salmonella typhimurium by regulating SPI-1 and flagella genes. PLoS One. (2012) 7:e37059. doi: 10.1371/journal.pone.0037059
60. Ritchie AJ, Jansson A, Stallberg J, Nilsson P, Lysaght P, Cooley MA. The Pseudomonas aeruginosa quorum-sensing molecule N-3-(oxododecanoyl)-Lhomoserine lactone inhibits T-cell differentiation and cytokine production by a mechanism involving an early step in T-cell activation. Infect Immun. (2005) 73:1648-55. doi: 10.1128/IAI.73.3.1648-1655.2005

61. Pritchard DI, Todd I, Brown A, Bycroft BW, Chhabra SR, Williams P, et al. Alleviation of insulitis and moderation of diabetes in NOD mice following treatment with a synthetic Pseudomonas aeruginosa signal molecule, N-(3oxododecanoyl)-L-homoserine lactone. Acta Diabetol. (2005) 42:119-22. doi: 10.1007/s00592-005-0190-2

62. Kendall MM, Sperandio V. Cell-to-cell signaling in E. coli and Salmonella. EcoSal Plus. (2014) 6:1-15. doi: 10.1128/ecosalplus.ESP-0002-2013

63. Kawasaki K, Ernst RK, Miller SI. 3-O-deacylation of lipid A by PagL, a PhoP/PhoQ-regulated deacylase of Salmonella typhimurium, modulates signaling through toll-like receptor 4. J Biol Chem. (2004) 279:20044-8. doi: 10.1074/jbc.M401275200

64. Verbrugghe E, Boyen F, van Parys A, van Deun K, Croubels S, Thompson A, et al. Stress induced Salmonella Typhimurium recrudescence in pigs coincides with cortisol induced increased intracellular proliferation in macrophages. Vet Res. (2011) 42:118. doi: 10.1186/1297-9716-42-118

65. Moreira ML, Dorneles EM, Soares RP, Magalhães CP, Costa-Pereira C, Lage AP, et al. Cross-reactivity of commercially available anti-human monoclonal antibodies with canine cytokines: establishment of a reliable panel to detect the functional profile of peripheral blood lymphocytes by intracytoplasmic staining. Acta Vet Scand. (2015) 57:51. doi: 10.1186/s13028-015-0142-y

66. Pullinger GD, Carnell SC, Sharaff FF, van Diemen PM, Dziva F, Morgan E, et al. Norepinephrine augments Salmonella enterica-induced enteritis in a manner associated with increased net replication but independent of the putative adrenergic sensor kinases QseC and QseE. Infect Immun. (2010) 78:372-80. doi: 10.1128/IAI.01203-09

67. Hald T, Wingstrand A, Swanenburg M, Altrock A, Thorberg BM. The occurrence and epidemiology of Salmonella in European pig slaughterhouses. Epidemiol Infect. (2003) 131:1187-203. doi: 10.1017/s0950268803001171

68. Meurens F, Summerfield A, Nauwynck H, Saif L, Gerdts V. The pig: a model for human infectious diseases. Trends Microbiol. (2012) 20:50-7. doi: 10.1016/ j.tim.2011.11.002

69. Mair KH, Sedlak C, Käser T, Pasternak A, Levast B, Gerner W, et al. The porcine innate immune system: an update. Dev Comp Immunol. (2014) 45:321-43. doi: 10.1016/j.dci.2014.03.022

70. Zhang Q, Widmer G, Tzipori S. A pig model of the human gastrointestinal tract. Gut Microbes. (2013) 4:193-200. doi: 10.4161/gmic.23867

71. Roura E, Koopmans S-J, Lallès J-P, Le Huerou-Luron I, Jager ND, Schuurman $\mathrm{T}$, et al. Critical review evaluating the pig as a model for human nutritional physiology. Nutr Res Rev. (2016) 29:60-90. doi: 10.1017/S095442241600 0020

Conflict of Interest: The authors declare that the research was conducted in the absence of any commercial or financial relationships that could be construed as a potential conflict of interest.

Copyright (c) 2020 Reiske, Schmucker, Steuber, Toulouse, Pfaffinger and Stefanski. This is an open-access article distributed under the terms of the Creative Commons Attribution License (CC BY). The use, distribution or reproduction in other forums is permitted, provided the original author(s) and the copyright owner(s) are credited and that the original publication in this journal is cited, in accordance with accepted academic practice. No use, distribution or reproduction is permitted which does not comply with these terms. 